ISSN 1855-3966 (printed edn.), ISSN 1855-3974 (electronic edn.)

ARS MATHEMATICA CONTEMPORANEA 21 (2021) \#P1.09

https://doi.org/10.26493/1855-3974.2488.f5c

(Also available at http://amc-journal.eu)

\title{
Nonlinear maps preserving the elementary symmetric functions
}

\author{
Constantin Costara \\ Faculty of Mathematics and Informatics, Ovidius University of Constanţa, \\ Mamaia Boul. 124, Constanţa, Romania
}

Received 24 November 2020, accepted 5 April 2021, published online 1 September 2021

\begin{abstract}
Let $\mathcal{M}_{n}$ be the algebra of all $n \times n$ complex matrices, and for a natural number $2 \leq$ $k \leq n$ denote by $E_{k}(x)$ the $k$ th elementary symmetric function on the eigenvalues of $x \in \mathcal{M}_{n}$. For two maps $\varphi, \psi: \mathcal{M}_{n} \rightarrow \mathcal{M}_{n}$, one of them being surjective, we prove that if $E_{k}(\lambda x+y)=E_{k}(\lambda \varphi(x)+\psi(y))$ for each $\lambda \in \mathbf{C}$ and $x, y \in \mathcal{M}_{n}$, then $\varphi=\psi$ on $\mathcal{M}_{n}$, the common value being a linear map from $\mathcal{M}_{n}$ into itself. In particular, for $3 \leq k \leq n$ the general form of $\varphi$ and $\psi$ can be computed explicitly.
\end{abstract}

Keywords: Elementary symmetric function, nonlinear, preserver.

Math. Subj. Class. (2020): 15A15, 15A86

\section{Introduction and statement of the result}

For a natural number $n$, let us denote by $\mathcal{M}_{n}$ the algebra of all $n \times n$ matrices over the complex field C. By $I_{n} \in \mathcal{M}_{n}$ we shall denote the $n \times n$ identity matrix. For $x \in \mathcal{M}_{n}$, by $\operatorname{tr}(x)$ we shall denote its usual trace, and by $\operatorname{det}(x)$ its determinant. Also, by $x^{t} \in \mathcal{M}_{n}$ we shall denote the transpose of $x$.

For $k \in\{1, \ldots, n\}$, a $k$-by- $k$ principal submatrix of $x \in \mathcal{M}_{n}$ is the submatrix of $x$ which lies in the rows and columns of $x$ indexed by $J \subseteq\{1, \ldots, n\}$ with $|J|=k$. Equivalently, we eliminate from the matrix $x$ the rows and the columns which are not in $J$. The determinant of the $k$-by- $k$ principal submatrix given by $J \subseteq\{1, \ldots, n\}$ is called a $k$-by- $k$ principal minor, and shall be denoted by $\Delta_{J}(x)$. There are $\left(\begin{array}{l}n \\ k\end{array}\right)$ different $k$-by- $k$ principal minors, and put

$$
E_{k}(x)=\sum_{|J|=k} \Delta_{J}(x) \quad\left(x \in \mathcal{M}_{n}, k=\overline{1, n}\right) .
$$

E-mail address: cdcostara@univ-ovidius.ro (Constantin Costara)

(ㄷ)(7) This work is licensed under https://creativecommons.org/licenses/by/4.0/ 
In particular, $k=1$ in (1.1) gives $E_{1}(x)=\operatorname{tr}(x)$ and $k=n$ gives $E_{n}(x)=\operatorname{det}(x)$ for each $x \in \mathcal{M}_{n}$.

For $k \in\{1, \ldots, n\}$, the $k$ th elementary symmetric function on the complex numbers $\lambda_{1}, \ldots, \lambda_{n}$ is

$$
S_{k}\left(\lambda_{1}, \ldots, \lambda_{n}\right)=\sum_{1 \leq i_{1}<\cdots<i_{k} \leq n} \prod_{j=1}^{k} \lambda_{i_{j}} .
$$

(We have a sum of $\left(\begin{array}{l}n \\ k\end{array}\right)$ products in (1.2).) For $x \in \mathcal{M}_{n}$, if the spectrum $\sigma(x)$ of $x$ (taking into account multiplicities) is $\left\{\alpha_{1}, \ldots, \alpha_{n}\right\}$, the equality

$$
\operatorname{det}\left(\lambda I_{n}+x\right)=\lambda^{n}+\lambda^{n-1} E_{1}(x)+\cdots+E_{n}(x) \quad\left(x \in \mathcal{M}_{n}, \lambda \in \mathbf{C}\right)
$$

implies that

$$
E_{k}(x)=S_{k}(\sigma(x)) \quad\left(x \in \mathcal{M}_{n}, k=\overline{1, n}\right) .
$$

Thus, for each $k \in \overline{1, n}$ and $x \in \mathcal{M}_{n}$ we have that $E_{k}(x)$ is the $k$ th elementary symmetric function on the eigenvalues of $x$.

Frobenius studied in [5] linear maps on $\mathcal{M}_{n}$ which preserve the determinant. He proved that if $\phi: \mathcal{M}_{n} \rightarrow \mathcal{M}_{n}$ is a bijective linear map such that $\operatorname{det}(\phi(x))=\operatorname{det}(x)$ for each $x \in \mathcal{M}_{n}$, there exist then invertible matrices $a, b \in \mathcal{M}_{n}$ satisfying $\operatorname{det}(a b)=1$ such that either $\phi(x)=a x b$ for each $x \in \mathcal{M}_{n}$, or $\phi(x)=a x^{t} b$ for each $x \in \mathcal{M}_{n}$. One way to generalize this result is to relax the linearity assumption on the map $\phi$. In [4], Dolinar and Šemrl proved that we arrive at the same conclusion if we merely suppose that $\phi: \mathcal{M}_{n} \rightarrow \mathcal{M}_{n}$ is a surjective map such that

$$
\operatorname{det}(\lambda x+y)=\operatorname{det}(\lambda \phi(x)+\phi(y)) \quad\left(x, y \in \mathcal{M}_{n}, \lambda \in \mathbf{C}\right) .
$$

(In this case, the linearity for the map $\phi$ is part of the conclusion.) Another way to generalize the result of Frobenius is to consider the elementary symmetric functions $E_{k}$ instead of the determinant. The following theorem was proved by Marcus and Purves in [7] for $4 \leq k<n$ and by Beasley in [1] for $3=k<n$.

Theorem 1.1 ([7, Theorem 3.1] and [1, Theorem 1.1]). Let $3 \leq k<n$ and let $\phi: \mathcal{M}_{n} \rightarrow$ $\mathcal{M}_{n}$ be a linear map such that

$$
E_{k}(x)=E_{k}(\phi(x)) \quad\left(x \in \mathcal{M}_{n}\right) .
$$

There exist then an invertible matrix $a \in \mathcal{M}_{n}$ and $\eta \in \mathbf{C}$ satisfying $\eta^{k}=1$ such that either

$$
\phi(x)=\operatorname{maxa}^{-1} \quad\left(x \in \mathcal{M}_{n}\right),
$$

or

$$
\phi(x)=\operatorname{\eta ax}^{t} a^{-1} \quad\left(x \in \mathcal{M}_{n}\right) .
$$

The result of Frobenius shows that the conclusion of Theorem 1.1 does not hold if $k=n$. The same happens if $k=1$ (see, for example, [6, Section 2]) or $k=2$ (see, for example, [7, Section 4] or [6, Section 3].) However, if the linear map $\phi: \mathcal{M}_{n} \rightarrow \mathcal{M}_{n}$ preserves both the trace and the determinant, then there exists an invertible matrix $a \in \mathcal{M}_{n}$ such that either

$$
\phi(x)=a x a^{-1} \quad\left(x \in \mathcal{M}_{n}\right)
$$


or

$$
\phi(x)=a x^{t} a^{-1} \quad\left(x \in \mathcal{M}_{n}\right) .
$$

(See, for example, [8, Theorem 1] or [9, Theorem 3].) Thus, if $E_{k}(x)=E_{k}(\phi(x))$ for each $k \in\{1, n\}$ and $x \in \mathcal{M}_{n}$, then $\phi$ is of the form given by (1.4) or (1.5). Also, if the linear $\operatorname{map} \phi: \mathcal{M}_{n} \rightarrow \mathcal{M}_{n}$ satisfies $E_{k}(x)=E_{k}(\phi(x))$ for each $k \in\{2, n\}$ and $x \in \mathcal{M}_{n}$, there exists then an invertible matrix $a \in \mathcal{M}_{n}$ such that either

$$
\phi(x)=\operatorname{\eta axa}^{-1} \quad\left(x \in \mathcal{M}_{n}\right),
$$

or

$$
\phi(x)=\operatorname{\eta ax}^{t} a^{-1} \quad\left(x \in \mathcal{M}_{n}\right),
$$

where $\eta=1$ if $n$ is odd, and $\eta=-1$ or $\eta=1$ if $n$ is even. (See, for example, [9, Theorem 4].)

The aim of this article is to improve the results of Theorem 1.1 in a way that is similar to [4, Theorem 1.1]. Thus, we eliminate the linearity assumption on the map $\phi$ and we impose a strengthened preservation condition which is suggested by (1.3). Incidentally, the result holds with a preservation condition which is stated for two maps $\varphi$ and $\psi$ on $\mathcal{M}_{n}$ instead of a single map $\phi$.

Theorem 1.2. Let $2 \leq k \leq n$ and consider two maps $\varphi, \psi: \mathcal{M}_{n} \rightarrow \mathcal{M}_{n}$, one of them being surjective, such that

$$
E_{k}(\lambda x+y)=E_{k}(\lambda \varphi(x)+\psi(y)) \quad\left(x, y \in \mathcal{M}_{n}, \lambda \in \mathbf{C}\right) .
$$

Then $\varphi=\psi$ on $\mathcal{M}_{n}$, the common value being a linear map of $\mathcal{M}_{n}$ into itself.

As a corollary, we obtain the following generalization of Theorem 1.1.

Corollary 1.3. Let $3 \leq k<n$ and consider two maps $\varphi, \psi: \mathcal{M}_{n} \rightarrow \mathcal{M}_{n}$, one of them being surjective, such that (1.8) holds. There exist then an invertible matrix $a \in \mathcal{M}_{n}$ and $\eta \in \mathbf{C}$ satisfying $\eta^{k}=1$ such that either

$$
\varphi(x)=\psi(x)=\operatorname{\eta axa}^{-1} \quad\left(x \in \mathcal{M}_{n}\right),
$$

or

$$
\varphi(x)=\psi(x)=\operatorname{\eta ax}^{t} a^{-1} \quad\left(x \in \mathcal{M}_{n}\right) .
$$

Of course, if we suppose that (1.8) holds for $k \in\{1, n\}$, then $\varphi=\psi$ on $\mathcal{M}_{n}$, the common value being a linear map of the form (1.4) or (1.5), and if we suppose that (1.8) holds for $k \in\{2, n\}$, then $\varphi=\psi$ on $\mathcal{M}_{n}$, the common value being a linear map of the form (1.6) or (1.7).

Since Theorem 1.2 also holds for $k=n$, we obtain a different proof for the following slight generalization of [4, Theorem 1.1]. (See also [2, Theorem 1] and [3, Theorem 1].)

Corollary 1.4. Consider two maps $\varphi, \psi: \mathcal{M}_{n} \rightarrow \mathcal{M}_{n}$, one of them being surjective, such that

$$
\operatorname{det}(\lambda x+y)=\operatorname{det}(\lambda \varphi(x)+\psi(y)) \quad\left(x, y \in \mathcal{M}_{n}, \lambda \in \mathbf{C}\right) .
$$

There exist then invertible matrices $a, b \in \mathcal{M}_{n}$ satisfying $\operatorname{det}(a b)=1$ such that either

$$
\varphi(x)=\psi(x)=a x b \quad\left(x \in \mathcal{M}_{n}\right),
$$

or

$$
\varphi(x)=\psi(x)=a x^{t} b \quad\left(x \in \mathcal{M}_{n}\right)
$$




\section{Preliminary lemmas}

Let $2 \leq k \leq n$. For $x, y \in \mathcal{M}_{n}$, consider the complex polynomial (with respect to $\lambda$ ) given by

$$
\lambda \mapsto E_{k}(\lambda x+y)
$$

This section is devoted to the study of these polynomials. As a general property, let us observe that its degree is always at most $k$, the coefficient of $\lambda^{k}$ being exactly $E_{k}(x)$. In particular, the degree of the polynomial is also bounded by the rank of the matrix $x$. Also, if we fix $x \in \mathcal{M}_{n}$, then the coefficient of $\lambda^{k-1}$ is linear with respect to $y \in \mathcal{M}_{n}$. Indeed, this comes from (1.1) and the fact that for $J \subseteq\{1, \ldots, n\}$ with $|J|=k$ we have

$$
\Delta_{J}(\lambda x+y)=\lambda^{k} \Delta_{J}(x)+\lambda^{k-1} \operatorname{tr}\left(\operatorname{adj}\left(x_{J}\right) y_{J}\right)+\cdots+\Delta_{J}(y),
$$

where $x_{J}$ (respectively, $y_{J}$ ) is the principal submatrix of $x$ (respectively, $y$ ) corresponding to $J$, and for $z \in \mathcal{M}_{k}$ by adj $(z) \in \mathcal{M}_{k}$ we have denoted the (classical) adjoint of the matrix $z$, obtained from its cofactors.

This coefficient will play an important role in our approach to prove Theorem 1.1, and shall be studied thoroughly in this section.

Lemma 2.1. Let $2 \leq k \leq n$, and let $1 \leq i, j \leq n$, with $i \neq j$. There exists then a matrix $x_{0} \in \mathcal{M}_{n}$ such that, for each $y \in \mathcal{M}_{n}$ we have that the coefficient of $\lambda^{k}$ for the polynomial $\lambda \mapsto E_{k}\left(\lambda x_{0}+y\right)$ is zero, and the coefficient of $\lambda^{k-1}$ for the same polynomial is $y_{i j}$.

Proof. Suppose, without lost of generality, that $i=1$ and $j=2$. Put then $J_{0}=\{1, \ldots, k\}$, and let

$$
x_{0}=\left[\begin{array}{cccc}
0 & 0 & 0 & 0 \\
-1 & 0 & & 0 \\
0 & & I_{k-2} & 0 \\
0 & 0 & 0_{n-k}
\end{array}\right] \in \mathcal{M}_{n}
$$

For $y \in \mathcal{M}_{n}$, let us observe that

$$
\begin{aligned}
\Delta_{J_{0}}\left(\lambda x_{0}+y\right) & =\operatorname{det}\left[\begin{array}{cccc}
y_{11} & y_{12} & \cdots & y_{1 k} \\
-\lambda+y_{21} & y_{22} & \cdots & y_{2 k} \\
\vdots & \vdots & \lambda I_{k-2}+\left(y_{s t}\right)_{3 \leq s, t \leq k} \\
y_{k 1} & y_{k 2} &
\end{array}\right] \\
& =0 \cdot \lambda^{k}+y_{12} \lambda^{k-1}+\cdots .
\end{aligned}
$$

Also, if $|J|=k$ and $J \neq J_{0}$, then the degree of $\lambda \mapsto \Delta_{J}\left(\lambda x_{0}+y\right)$ with respect to $\lambda$ is at most $k-2$, since we have at most $k-2$ appearances of $\lambda$ in the principal submatrix of $\lambda x_{0}+y$ corresponding to $J$. We use now (1.1) to finish the proof.

The remaining of this section is devoted to prove that the same type of statement as the one of Lemma 2.1 also holds for $i=j$. This requires a little extra work.

Lemma 2.2. Let $2 \leq k \leq n$, and let $J_{0} \subseteq\{1, \ldots, n\}$ with $\left|J_{0}\right|=k-1$. There exists then a matrix $x_{0} \in \mathcal{M}_{n}$ such that, for each $y \in \mathcal{M}_{n}$ we have that the coefficient of $\lambda^{k}$ for the polynomial $\lambda \mapsto E_{k}\left(\lambda x_{0}+y\right)$ is zero, and the coefficient of $\lambda^{k-1}$ for the same polynomial is $\sum_{j \notin J_{0}} y_{j j}$. 
Proof. Suppose, without lost of generality, that $J_{0}=\{1, \ldots, k-1\}$. Let then

$$
x_{0}=\left[\begin{array}{cc}
I_{k-1} & 0 \\
0 & 0_{n-k+1}
\end{array}\right] \in \mathcal{M}_{n} .
$$

For $y \in \mathcal{M}_{n}$, let us observe that for each $j \in\{1, \ldots, n\} \backslash J_{0}$ we have that

$$
\Delta_{J_{0} \cup\{j\}}\left(\lambda x_{0}+y\right)=0 \cdot \lambda^{k}+y_{j j} \lambda^{k-1}+\cdots .
$$

Also, if $|J|=k$ and $\left|J \cap J_{0}\right| \leq k-2$, then the degree of $\lambda \mapsto \Delta_{J}\left(\lambda x_{0}+y\right)$ with respect to $\lambda$ is at most $k-2$, since we have at most $k-2$ appearances of $\lambda$ in the principal submatrix of $\lambda x_{0}+y$ corresponding to $J$. We use again (1.1) to finish the proof.

Corollary 2.3. Let $2 \leq k \leq n$, and let $1 \leq i, j \leq n$, with $i \neq j$. There exist then matrices $x_{1}, x_{2} \in \mathcal{M}_{n}$ such that, for each $y \in \mathcal{M}_{n}$ we have that the coefficient of $\lambda^{k}$ for the polynomial $\lambda \mapsto E_{k}\left(\lambda x_{1}+y\right)-E_{k}\left(\lambda x_{2}+y\right)$ is zero, and the coefficient of $\lambda^{k-1}$ for the same polynomial is $y_{j j}-y_{i i}$.

Proof. Consider $J \subseteq\{1, \ldots, n\} \backslash\{i, j\}$ such that $|J|=k-2$. We apply then Lemma 2.2 to $J \cup\{i\}$ to find a matrix $x_{1} \in \mathcal{M}_{n}$ such that, for each $y \in \mathcal{M}_{n}$ we have that the coefficient of $\lambda^{k}$ for the polynomial $\lambda \mapsto E_{k}\left(\lambda x_{1}+y\right)$ is zero, and the coefficient of $\lambda^{k-1}$ for the same polynomial is $\sum_{t \notin(J \cup\{i\})} y_{t t}$, and we apply the same lemma to $J \cup\{j\}$ to find a matrix $x_{2} \in \mathcal{M}_{n}$ such that, for each $y \in \mathcal{M}_{n}$ we have that the coefficient of $\lambda^{k}$ for the polynomial $\lambda \mapsto E_{k}\left(\lambda x_{2}+y\right)$ is zero, and the coefficient of $\lambda^{k-1}$ for the same polynomial is $\sum_{t \notin(J \cup\{j\})} y_{t t}$. To finish the proof, observe that $\left(\sum_{t \notin(J \cup\{i\})} y_{t t}\right)-\left(\sum_{t \notin(J \cup\{j\})} y_{t t}\right)=$ $y_{j j}-y_{i i}$.

Lemma 2.4. Let $2 \leq k \leq n$, and let $j \in\{1, \ldots, n\}$. There exist then $q>0, m, p \geq 0$ in $\mathbf{Z}$ and matrices $x_{0}, x_{1}, . ., x_{m+p} \in \mathcal{M}_{n}$ such that, for each $y \in \mathcal{M}_{n}$ we have that the coefficient of $\lambda^{k}$ for the polynomial

$$
\lambda \mapsto \sum_{i=0}^{m} E_{k}\left(\lambda x_{i}+y\right)-\left(\sum_{i=m+1}^{m+p} E_{k}\left(\lambda x_{i}+y\right)\right)
$$

is zero, and the coefficient of $\lambda^{k-1}$ for the same polynomial is $q \cdot y_{j j}$.

Proof. Consider $J \subseteq\{1, \ldots, n\} \backslash\{j\}$ such that $|J|=k-1$. We apply Lemma 2.2 to $J$ to find a matrix $x_{0} \in \mathcal{M}_{n}$ such that, for each $y \in \mathcal{M}_{n}$ we have that the coefficient of $\lambda^{k}$ for the polynomial $\lambda \mapsto E_{k}\left(\lambda x_{0}+y\right)$ is zero, and the coefficient of $\lambda^{k-1}$ for the same polynomial is $\sum_{t \notin J} y_{t t}=y_{j j}+\sum_{t \notin(J \cup\{j\})} y_{t t}$. For each $t \notin(J \cup\{j\})$ in $\{1, \ldots, n\}$, we apply Corollary 2.3 to $t \neq j$ to find matrices $x_{t}^{(1)}, x_{t}^{(2)} \in \mathcal{M}_{n}$ such that, for each $y \in \mathcal{M}_{n}$ we have that the coefficient of $\lambda^{k}$ for the polynomial $\lambda \mapsto E_{k}\left(\lambda x_{t}^{(1)}+y\right)-E_{k}\left(\lambda x_{t}^{(2)}+y\right)$ is zero, and the coefficient of $\lambda^{k-1}$ for the same polynomial is $y_{j j}-y_{t t}$. To finish the proof, observe that

$$
\sum_{t \notin J} y_{t t}+\left(\sum_{t \notin(J \cup\{j\})}\left(y_{j j}-y_{t t}\right)\right)=q \cdot y_{j j},
$$

for some strictly positive integer $q$. 


\section{Proof of the main result}

Let $2 \leq k \leq n$. Let us first observe that if $\varphi, \psi: \mathcal{M}_{n} \rightarrow \mathcal{M}_{n}$ satisfy (1.8), dividing by $\lambda \in \mathbf{C} \backslash\{0\}$ we obtain that $E_{k}(x+\mu y)=E_{k}(\varphi(x)+\mu \psi(y))$ for all $x, y \in \mathcal{M}_{n}$ and $\mu \in \mathbf{C} \backslash\{0\}$. By continuity, the same holds for $\mu=0$, too. Thus

$$
E_{k}(x+\mu y)=E_{k}(\varphi(x)+\mu \psi(y)) \quad\left(x, y \in \mathcal{M}_{n}, \mu \in \mathbf{C}\right) .
$$

That is, the same type of equalities as the ones in (1.8) hold, with the role of $\varphi$ and $\psi$ interchanged. Thus, without lost of generality, we may suppose for the remaining of the paper that the map $\varphi$ is surjective. (If not, then $\psi$ must be surjective, and we work with (3.1) instead of (1.8).)

Another immediate observation is the fact that if $\varphi$ and $\psi$ satisfy (1.8), for $\lambda=0$ in (1.8) and $\mu=0$ in (3.1) we see that $E_{k}(y)=E_{k}(\psi(y))$ for all $y \in \mathcal{M}_{n}$, respectively $E_{k}(x)=E_{k}(\varphi(x))$ for all $x \in \mathcal{M}_{n}$.

As a corollary of Lemma 2.1 and Lemma 2.4, the following result holds.

Theorem 3.1. Suppose $2 \leq k \leq n$, and let $i, j \in\{1, \ldots, n\}$. There exist then a nonzero scalar $\alpha$, positive integers $m$ and $p$ and matrices $x_{0}, x_{1}, . ., x_{m+p} \in \mathcal{M}_{n}$ such that, for each $y \in \mathcal{M}_{n}$ we have that the coefficient of $\lambda^{k}$ for the polynomial

$$
\lambda \mapsto \sum_{s=0}^{m} E_{k}\left(\lambda x_{s}+y\right)-\left(\sum_{s=m+1}^{m+p} E_{k}\left(\lambda x_{s}+y\right)\right)
$$

is zero, and the coefficient of $\lambda^{k-1}$ for the same polynomial is $\alpha \cdot y_{i j}$.

As a direct corollary of Theorem 3.1, we obtain the following test for the equality to $0 \in \mathcal{M}_{n}$ in terms of the functions $E_{k}$. (See also [7, Lemma 3.1].)

Corollary 3.2. Suppose $2 \leq k \leq n$. Let $y \in \mathcal{M}_{n}$ such that

$$
E_{k}(x+y)=E_{k}(x) \quad\left(x \in \mathcal{M}_{n}\right) .
$$

Then $y=0$.

Proof. Observe that (3.2) gives

$$
E_{k}(\lambda x+y)=\lambda^{k} E_{k}(x) \quad\left(x \in \mathcal{M}_{n}, \lambda \in \mathbf{C}\right) .
$$

Let $i, j \in\{1, \ldots, n\}$. By Theorem 3.1, there exist $\alpha \neq 0$, positive integers $m$ and $p$ and matrices $x_{0}, x_{1}, . ., x_{m+p} \in \mathcal{M}_{n}$ such that, for all $\lambda \in \mathbf{C}$,

$$
\sum_{s=0}^{m} E_{k}\left(\lambda x_{s}+y\right)-\left(\sum_{s=m+1}^{m+p} E_{k}\left(\lambda x_{s}+y\right)\right)=0 \cdot \lambda^{k}+\left(\alpha y_{i j}\right) \cdot \lambda^{k-1}+\cdots .
$$

Using (3.3), for all $\lambda \in \mathbf{C}$ we have that

$$
\begin{aligned}
\sum_{s=0}^{m} E_{k}\left(\lambda x_{s}+y\right)-\left(\sum_{s=m+1}^{m+p} E_{k}\left(\lambda x_{s}+y\right)\right) & =\lambda^{k}\left(\sum_{s=0}^{m} E_{k}\left(x_{s}\right)-\left(\sum_{s=m+1}^{m+p} E_{k}\left(x_{s}\right)\right)\right) \\
& =0 .
\end{aligned}
$$

Thus $\alpha y_{i j}=0$, and therefore $y_{i j}=0$. Since this holds for any $i$ and $j$, we obtain that $y=0 \in \mathcal{M}_{n}$. 
Theorem 3.1 gives us also linearity for the maps $\varphi$ and $\psi$ from the statement of Theorem 1.1.

Proof of Theorem 1.1. Let us prove first that (1.8) and the surjectivity of $\varphi$ implies that $\psi$ is linear on $\mathcal{M}_{n}$. To see this, consider $i, j \in\{1, \ldots, n\}$ and let us prove that $\psi_{i j}: \mathcal{M}_{n} \rightarrow \mathbf{C}$ is linear, where $\psi_{i j}$ is the $(i, j)$ entry of the map $\psi$. By Theorem 3.1, there exist $\alpha \neq 0$ in $\mathbf{C}$, natural numbers $m$ and $p$ and matrices $x_{0}, x_{1}, . ., x_{m+p} \in \mathcal{M}_{n}$ such that, for each $y \in \mathcal{M}_{n}$ we have that the coefficient of $\lambda^{k}$ for the polynomial

$$
\lambda \mapsto \sum_{s=0}^{m} E_{k}\left(\lambda x_{s}+y\right)-\left(\sum_{s=m+1}^{m+p} E_{k}\left(\lambda x_{s}+y\right)\right)
$$

is zero, and the coefficient of $\lambda^{k-1}$ for the same polynomial is $\alpha y_{i j}$. Since $\varphi$ is supposed surjective, let $w_{0}, w_{1}, \ldots, w_{m+p} \in \mathcal{M}_{n}$ such that $\varphi\left(w_{j}\right)=x_{j}$ for $j=0, \ldots, m+p$. Then for each $z \in \mathcal{M}_{n}$, we have that the coefficient of $\lambda^{k}$ for the polynomial

$$
\lambda \mapsto \sum_{s=0}^{m} E_{k}\left(\lambda \varphi\left(w_{s}\right)+\psi(z)\right)-\left(\sum_{s=m+1}^{m+p} E_{k}\left(\lambda \varphi\left(w_{s}\right)+\psi(z)\right)\right)
$$

is zero, and the coefficient of $\lambda^{k-1}$ for the same polynomial is $\alpha \psi_{i j}(z)$. Using (1.8), for all $\lambda \in \mathbf{C}$ we have that

$$
\sum_{s=0}^{m} E_{k}\left(\lambda \varphi\left(w_{s}\right)+\psi(z)\right)-\left(\sum_{s=m+1}^{m+p} E_{k}\left(\lambda \varphi\left(w_{s}\right)+\psi(z)\right)\right)
$$

equals

$$
\sum_{s=0}^{m} E_{k}\left(\lambda w_{s}+z\right)-\left(\sum_{s=m+1}^{m+p} E_{k}\left(\lambda w_{s}+z\right)\right) .
$$

The remark at the beginning of Section 2 shows that the coefficient of $\lambda^{k-1}$ for the polynomial $\lambda \mapsto \sum_{s=0}^{m} E_{k}\left(\lambda w_{s}+z\right)-\left(\sum_{s=m+1}^{m+p} E_{k}\left(\lambda w_{s}+z\right)\right)$ is linear with respect to $z \in \mathcal{M}_{n}$. Therefore, $\psi_{i j}$ is linear with respect to $z \in \mathcal{M}_{n}$.

Thus $\psi: \mathcal{M}_{n} \rightarrow \mathcal{M}_{n}$ is linear and $E_{k}(x)=E_{k}(\psi(x))$ for each $x \in \mathcal{M}_{n}$. If $\psi(y)=0$, then for each $x \in \mathcal{M}_{n}$ we have that

$$
\begin{aligned}
E_{k}(x) & =E_{k}(\psi(x))=E_{k}(\psi(x)+\psi(y))=E_{k}(\psi(x+y)) \\
& =E_{k}(x+y) .
\end{aligned}
$$

Then Corollary 3.2 gives $y=0$. Thus the linear map $\psi$ is injective on $\mathcal{M}_{n}$, and therefore bijective. Using (1.8), the linearity of $\psi$ and the fact that $E_{k}(z)=E_{k}\left(\psi^{-1}(z)\right)$ for each $z$, then for each $x, y \in \mathcal{M}_{n}$ we have that

$$
\begin{aligned}
E_{k}(x+y) & =E_{k}(\varphi(x)+\psi(y))=E_{k}\left(\psi^{-1}(\varphi(x)+\psi(y))\right) \\
& =E_{k}\left(\left(\psi^{-1} \circ \varphi\right)(x)+y\right) .
\end{aligned}
$$

Denoting $z=x+y$, we conclude that $E_{k}(z)=E_{k}\left(\left(\left(\psi^{-1} \circ \varphi\right)(x)-x\right)+z\right)$ for each $x, z \in \mathcal{M}_{n}$. Then Corollary 3.2 gives $\left(\psi^{-1} \circ \varphi\right)(x)-x=0$, equality which holds for every $x \in \mathcal{M}_{n}$. Thus $\varphi=\psi$ on $\mathcal{M}_{n}$. 


\section{ORCID iDs}

Constantin Costara (iD https://orcid.org/0000-0001-7621-5591

\section{References}

[1] L. B. Beasley, Linear transformations on matrices: The invariance of the third elementary symmetric function, Canadian J. Math. 22 (1970), 746-752, doi:10.4153/cjm-1970-084-x.

[2] C. Costara, Nonlinear determinant preserving maps on matrix algebras, Linear Algebra Appl. 583 (2019), 165-170, doi:10.1016/j.laa.2019.09.003.

[3] C. Costara, Nonlinear invertibility preserving maps on matrix algebras, Linear Algebra Appl. 602 (2020), 216-222, doi:10.1016/j.laa.2020.05.010.

[4] G. Dolinar and P. Šemrl, Determinant preserving maps on matrix algebras, Linear Algebra Appl. 348 (2002), 189-192, doi:10.1016/s0024-3795(01)00578-x.

[5] G. Frobenius, Über die darstellung der endlichen gruppen durch lineare substitutionen, Deutsch. Akad. Wiss. (1897), 994-1015, doi:10.3931/e-rara-18879.

[6] A. Kovacs, Trace preserving linear transformations on matrix algebras, Linear and Multilinear Algebra 4 (1976/77), 243-250, doi:10.1080/03081087708817158.

[7] M. Marcus and R. Purves, Linear transformations on algebras of matrices: the invariance of the elementary symmetric functions, Canadian J. Math. 11 (1959), 383-396, doi:10.4153/ cjm-1959-039-4.

[8] H. Minc, Linear transformations on nonnegative matrices, Linear Algebra Appl. 9 (1974), 149153, doi:10.1016/0024-3795(74)90033-0.

[9] H. Minc, The invariance of elementary symmetric functions, Linear and Multilinear Algebra 4 (1976), 209-215, doi:10.1080/03081087608817153. 